\title{
The links between prenatal stress and offspring development and psychopathology: disentangling environmental and inherited influences
}

\author{
F. Rice ${ }^{1 *}$, G. T. Harold ${ }^{2}$, J. Boivin ${ }^{2}$, M. van den Bree ${ }^{1}$, D. F. Hay ${ }^{2}$ and A. Thapar $^{1}$ \\ ${ }^{1}$ Department of Psychological Medicine, School of Medicine, Cardiff University, Cardiff, UK \\ ${ }^{2}$ School of Psychology, Cardiff University, Cardiff, UK
}

Background. Exposure to prenatal stress is associated with later adverse health and adjustment outcomes. This is generally presumed to arise through early environmentally mediated programming effects on the foetus. However, associations could arise through factors that influence mothers' characteristics and behaviour during pregnancy which are inherited by offspring.

Method. A 'prenatal cross-fostering' design where pregnant mothers are related or unrelated to their child as a result of in vitro fertilization (IVF) was used to disentangle maternally inherited and environmental influences. If links between prenatal stress and offspring outcome are environmental, association should be observed in unrelated as well as related mother-child pairs. Offspring birth weight and gestational age as well as mental health were the outcomes assessed.

Results. Associations between prenatal stress and offspring birth weight, gestational age and antisocial behaviour were seen in both related and unrelated mother-offspring pairs, consistent with there being environmental links. The association between prenatal stress and offspring anxiety in related and unrelated groups appeared to be due to current maternal anxiety/depression rather than prenatal stress. In contrast, the link between prenatal stress and offspring attention deficit hyperactivity disorder was only present in related mother-offspring pairs and therefore was attributable to inherited factors.

Conclusions. Genetically informative designs can be helpful in testing whether inherited factors contribute to the association between environmental risk factors and health outcomes. These results suggest that associations between prenatal stress and offspring outcomes could arise from inherited factors and post-natal environmental factors in addition to causal prenatal risk effects.

Received 24 November 2008; Revised 11 March 2009; Accepted 21 March 2009; First published online 29 May 2009

Key words: ADHD, anxiety, birth weight, child, conduct.

\section{Introduction}

Experimental and prospective epidemiological research has highlighted the possibility that prenatal maternal stress and anxiety could have long-lasting effects on the physical and psychological development of offspring (Huizink et al. 2004; Rice et al. 2007; Talge et al. 2007). There is now much evidence from prospective surveys showing that the offspring of mothers with high levels of anxiety during mid to late pregnancy are more likely to have both emotional and

* Address for correspondence: F. Rice, Ph.D., Division of Psychology and Language Sciences, University College London, 26 Bedford Way, London WC1H 0AP, UK.

(Email: f.rice@ucl.ac.uk)

Portions of this paper were presented at the Behaviour Genetics Association, Amsterdam, 5 June 2007. disruptive behaviour problems as children ( $\mathrm{O}^{\prime}$ Connor et al. 2003; Huizink et al. 2004; van den Bergh \& Marcoen, 2004). Prenatal depression and anxiety have also been reported to be associated with premature onset of labour and reductions in birth weight in naturalistic studies (Rahman et al. 2007). Prospective studies tend to identify stress during late pregnancy as showing the strongest associations with offspring psychopathology and birth weight (Rice et al. 2007; Talge et al. 2007). Potential mechanisms proposed to explain these associations include effects of stress on placental blood flow and maternal glucocorticoid effects on foetal brain development that may be mediated through early programming effects (Welberg \& Seckl, 2001).

Proving that prenatal stress has true risk effects on offspring outcomes is, however, challenging. One 
important consideration is that associations between prenatal stress/anxiety and offspring outcomes may be attributable to post-natal maternal anxiety and depression (O'Connor et al. 2002a,b; Barrett, 2006). Another problem is that exposure to the maternally provided prenatal environment is not random and many important prenatal factors that impact on offspring development in utero are influenced by heritable maternal characteristics (Rutter et al. 2001; Maughan et al. 2004; Kendler \& Prescott, 2006). Thus it is possible that the associations observed in epidemiological studies could be accounted for by inherited pathways, whereby heritable factors influence both maternal predisposition to experiencing stress/ anxiety and offspring outcomes. However, in humans, such effects are difficult to rule out without the use of experimental or genetically informative designs (Rutter, 2007) and prospective epidemiological studies are not able to address this issue.

Prenatal cross-fostering of animals allows the maternally provided prenatal environment and inherited factors to be disentangled. Previously, it has not been possible to assess human offspring whose prenatal environment is provided by a biologically unrelated mother. This is now feasible because of the increased use of in vitro fertilization (IVF) as a means of conception: IVF has resulted in over 100000 children worldwide (Adamson et al. 2006) and 1.3-3.6\% of European births (Nygren \& Andersen, 1999). Children conceived via these methods can be related to both parents (homologous IVF), the mother only (IVF with sperm donation), the father only (IVF with egg donation) or to neither parent (IVF with embryo donation). With egg and embryo donation, the mother provides the intra-uterine environment but is not related to the child (Thapar et al. 2007).

Assembling a sample of 779 children born through IVF, we set out to examine offspring whose prenatal environment was provided by a related mother $(n=$ 574) and the rarer group of children where it was provided by an unrelated mother $(n=205)$. This research design allows maternally provided inherited factors to be separated from prenatal influences (Rice et al. 2009). The aim was to test whether previously observed associations between prenatal stress and offspring outcomes are accounted for by maternally provided inherited factors. We focused on links between maternally reported prenatal stress with offspring: (1) birth weight; (2) gestational age ; (3) anxiety symptoms; (4) antisocial behaviour; and (5) attention deficit hyperactivity disorder (ADHD) symptoms. These offspring outcomes were selected because associations with prenatal stress have been reported but it is unclear if these are attributable to inherited or prenatal environmental processes or both.

\section{Method}

Sample

Families who had a live birth between 1994 and 2002 (children aged 4-10 years) following successful IVF treatment from any of the four conception groups described were recruited from 19 UK clinics and one US clinic. Gamete donors were unrelated to either parent. Initial contact was made through clinic staff. Data were collected through postal questionnaire and, where consent was provided, review of antenatal records. In this cross-sectional analysis, mothers and fathers reported on their own and their child's health. For multiple births, parents reported on the first-born twin $(n=161)$ or triplet $(n=18)$ only. Data were available from 526 fathers and 779 mothers. For this report we required a reply from the mother who reported on prenatal complications and prenatal stress. Questionnaire data from 779 families were available; 387 homologous IVF (parents' own gametes used), 187 sperm donation, 170 oocyte donation and 35 embryo donation. The majority of women gave written consent for their antenatal records to be reviewed $(77 \%$, $n=585$ ) although we did not request records for mothers who were treated in the US clinic $(n=23)$. For mothers treated at the $19 \mathrm{UK}$ clinics, antenatal records were not obtainable for a number of women because of difficulties in locating the notes. Thus, for this analysis, we had medical record data for $474(81 \%)$ of the 585 mothers who gave consent.

\section{Measures}

\section{Antenatal predictor variables}

Prenatal stress. This was assessed using an 11-point Likert scale (range 0-10; ' completely relaxed, no stress or worry at all' to 'as stressed or worried as you can possibly imagine'). As prior research suggests early and late prenatal stress has different effects, mothers retrospectively reported on stress during early (0-16 weeks), mid (17-30 weeks) and late (31-40 weeks) stages of pregnancy. Mothers were asked to circle the appropriate point on the scale that best described how they felt at each of those stages of pregnancy. On the basis of previous research (Rice et al. 2007; see below) we focused on late prenatal stress.

Reliability and validity of prenatal stress measure. We examined test-retest reliability in a subsample of this cohort: 94 women completed the measure on two occasions approximately 3 or 4 years apart: intra-class correlation coefficients (ICC) were high (early $\mathrm{ICC}=0.737$, mid ICC $=0.726$, late $\mathrm{ICC}=0.705)$, illustrating high stability of reporting in this sample. 
Appendix 1 shows the bivariate test-retest correlations for perceived stress between different stages of pregnancy. The cross-stage test-retest correlations were lower than the within-stage correlations. There was evidence that mothers were able to distinguish early and late pregnancy stress, as the product moment correlation between the two scores was not significant $(r=0.180, p=0.09)$. We tested the validity of the measure by examining associations with prenatal stress and perinatal outcomes from medical records. Studies that have been able to use more objective measures of prenatal stress (such as epidemiological studies of disasters, assays of hypothalamic-pituitaryadrenal axis activity and experimental animal studies) have shown that late pregnancy stress is associated with prematurity and reduced size at birth (Wadhwa et al. 1993; Sandman et al. 1997; Chang et al. 2002; Lederman et al. 2004; Weinstock, 2005). Thus, if our subjective and retrospective measure of prenatal stress shows construct validity it should also show association with these outcomes. Maternal report of late pregnancy stress was associated with reduced birth length $(r=-0.214, p=0.001)$, reduced head circumference $(r=-0.133, p=0.009)$, reduced birth weight $(r=-0.244, p=0.001)$, reduced gestational age $(r=-0.273, p=0.001)$ and prematurity (gestation $<37$ weeks) (odds ratio $=1.24, p=0.005$ ). This pattern of results therefore suggests that this measure shows good construct validity in that it shows significant association with multiple objective measures of infant size at birth.

\section{Offspring outcomes}

Birth weight. Agreement between maternal reports and medical records for birth weight was nearly perfect in the present sample $(r=0.985, p=0.001)$. This allowed us to use the full sample of maternal reports rather than the subsample with antenatal records available.

Gestational age. Mothers reported the week of pregnancy during which their child was born. Agreement with information in antenatal records was excellent $(r=0.959, p=0.001)$. Again, this allowed us to use the full sample of maternal reports.

\section{Offspring psychopathology}

Child anxiety. Mothers reported on their child's anxiety levels in the previous 3 months by completing a sixitem checklist of DSM-IV (APA, 1994) symptoms of generalized anxiety disorder where each item was rated on a three-point scale. Sample items included nervous, highly strung or tense, worries, easily tired.
Child antisocial behaviour. Mothers and fathers completed the Strengths and Difficulties Questionnaire (SDQ; Goodman, 1997) about their child's behaviour. Antisocial behaviour was assessed with the conduct problems scale of the SDQ. We chose to combine maternal and paternal reports in this way as it is recommended for defining childhood antisocial behaviour (Whittinger et al. 2007). If either the mother or the father endorsed a symptom, it was counted as present.

Child ADHD symptoms. Mothers completed the 14-item Du Paul questionnaire (Du Paul, 1981) about their child's symptoms of ADHD.

Current maternal anxiety/depression. Mothers reported on their current anxiety and depression symptoms using the Hospital Anxiety and Depression Scale (Zigmond \& Snaith, 1983).

\section{Covariates}

Covariates were included in each analysis depending on the outcome variable. Covariates were selected on the basis of known correlates of the independent and dependent variables and patterns of association observed in the present sample. Intercorrelations between all covariates and independent and dependent variables are shown in Table 2 . We included child age, gender and family social class as covariates where offspring psychopathology was an outcome variable. For analyses of birth weight as an outcome variable, we included maternal age at childbirth, maternal height, child gender and social class as covariates since these have been associated with birth weight in the literature (Valero de Bernabe et al. 2004). In addition, the following antenatal factors were adjusted for, either because of an association with levels of prenatal stress, or a reported link with infant birth weight in the literature (Wadhwa et al. 1998; Valero de Bernabe et al. 2004): vaginal bleeding; admission to hospital for high blood pressure/oedema; maternal prenatal cigarette smoking (yes/no); maternal prenatal alcohol use (yes/no); and infant plurality. All of these antenatal factors were coded as binary variables $(0 / 1)$ and a composite was computed and entered into the regression model.

\section{Methodology and statistics}

The key step was to test for association between prenatal stress and child outcome in the group where the mother experiencing the pregnancy was unrelated to the child $(n=205)$. If there is association, then it cannot be due to inherited influences on maternal behaviour 
Table 1. Descriptive statistics

\begin{tabular}{lllll}
\hline & $\begin{array}{l}\text { Mother related } \\
\text { to foetus, mean (s.D.) }\end{array}$ & $\begin{array}{l}\text { Mother unrelated } \\
\text { to foetus, mean (s.D.) }\end{array}$ & $\begin{array}{l}\text { Statistical } \\
\text { test }\end{array}$ & Significance: $p$ \\
\hline Child birth weight, g & $3083.14(677.08)$ & $3046.46(735.72)$ & $t=0.66$ & 0.51 \\
Mean family income range, US\$ & $60000-80000$ & $60000-80000$ & Kendall's $\tau=0.08$ & $0.04^{*}$ \\
Socio-occupational classification of & 19 & 14 & $\chi^{2}=1.76$ & 0.19 \\
$\quad$ main earner, \% operative/elementary & & & & \\
Main earner unemployed, \% & 4 & 4 & $\chi^{2}=0.88$ & 0.77 \\
Child antisocial behaviour & $1.78(1.50)$ & $2.06(1.59)$ & $t=2.25$ & $0.03^{*}$ \\
Child anxiety & $2.34(2.45)$ & $2.08(2.02)$ & $t=-1.34$ & 0.18 \\
Child ADHD symptoms & $10.20(7.54)$ & $11.38(7.30)$ & $t=1.92$ & 0.06 \\
Child age, years & $6.82(1.27)$ & $6.47(1.29)$ & $t=3.41$ & $0.001^{* *}$ \\
Mother anxiety & $7.12(3.73)$ & $7.53(3.90)$ & $t=1.33$ & 0.19 \\
Mother depression & $3.67(2.66)$ & $3.99(2.59)$ & $t=1.46$ & 0.14 \\
Mother late prenatal stress & $3.57(2.68)$ & $4.12(2.95)$ & $t=2.41$ & $0.02^{*}$ \\
Mother age at pregnancy, years & $34.06(3.63)$ & $38.92(6.02)$ & $t=13.68$ & $0.001^{* *}$ \\
Singleton births, \% & 77.6 & 76.3 & $\chi^{2}=0.13$ & 0.72 \\
High blood pressure/oedema, \% & 24.8 & 11.3 & $\chi^{2}=21.25$ & $0.001^{* *}$ \\
Prenatal smoking, \% & 4.4 & 6.5 & $\chi^{2}=1.16$ & 0.28 \\
Prenatal alcohol use, yes/no & 25.0 & 19.6 & $\chi^{2}=2.43$ & 0.12 \\
\hline
\end{tabular}

S.D., Standard deviation; ADHD, attention-deficit hyperactivity disorder.

${ }^{*} p<0.05,{ }^{* *} p<0.01$.

and child outcome since mother and child are biologically unrelated. The second step was to compare the magnitude of association between prenatal stress and outcome in the related and unrelated groups. When association is only seen in the related group, this is indicative of transmission due to inherited factors. When correlation is seen in both groups, but is greater in the related group, this is indicative of both inherited and prenatal environmental transmission. Multiple regression analysis was used to test for associations between prenatal stress and offspring outcomes separately in the related and unrelated groups. Raw scores were analysed, as the outcome variables were approximately normally distributed (skewness $<1$ ). Covariates were included as predictor variables in the regression model. To test for gender differences, analyses were run separately for boys and girls. Analyses predicting birth weight were repeated restricting the analysis to singleton births.

When associations between prenatal stress and offspring outcomes were detected in each group, interactions were tested using a regression framework. The whole sample was analysed and predictors included (1) the interaction term between prenatal stress and relatedness group and (2) the main effects of prenatal stress, antenatal complications and relatedness group. This interaction term measures the difference in association between prenatal stress and child outcome across the two offspring groups (related; unrelated). A significant interaction term indicates that the association between prenatal adversity and child outcome differs significantly according to group. Therefore, a significant interaction term is expected if inherited factors explain the association between prenatal stress and child outcome. However, it should be noted that a non-significant interaction term does not preclude the possibility that genetic factors contribute to an association. As outlined above, the relative importance of genetic and environmental factors can be inferred by simply examining the correlation coefficients for the related and unrelated groups separately.

When results suggested that association may be due to environmental factors (i.e. significant association in unrelated and related groups) we additionally examined whether this was attributable to current maternal anxiety/depression by including this variable as an additional covariate. Where appropriate, we tested for mediation effects of prenatal stress by current maternal anxiety/depression using the approach outlined by Baron \& Kenny (1986). Significance tests of mediation were calculated using the Aroian version of the Sobel test (Preacher \& Leonardelli, 2001).

\section{Results \\ Descriptives}

Table 1 shows descriptive characteristics of the sample and differences between the groups. The mean age 
of the children was 6.73 years (range 4-10 years, $51 \%$ male). Children in the egg and embryo donation groups were on average 5 months younger than those in the homologous and sperm donation groups. The mean current age for mothers was 42 years (range 29-62 years) and was 45 years (range 21-76 years) for fathers. The children were primarily of White/European descent (91.5\%). Socio-occupational status was assigned according to the occupation of the main earner (Office for National Statistics, 2000). There were no significant differences between participants recruited from the US clinic compared with the rest of the sample. The only exception was that US families reported a higher family income (Kendall's $\tau$ coefficient $=0.052, p=0.001$ ) (details available from F.R.).

In terms of mother and child adjustment, the present sample did not differ substantially from normative comparison data although there were some slight differences between conception groups (Shelton et al. in press). For obstetric factors, women in the egg and embryo donation groups were significantly older at conception $[F(3,782)=66.56, p=0.001]$ and more likely to report high blood pressure during pregnancy $\left[\chi^{2}(3)=23.78, p=0.001\right]$. However, there were no significant group differences in terms of smoking and alcohol use or the proportion of multiple births (Table 1). Table 2 shows the correlation matrix for all study variables including covariates and perinatal factors.

\section{Prenatal stress}

We first simultaneously entered prenatal stress during each stage to predict offspring outcomes separately in the related and unrelated groups. This identified stress during late pregnancy as having the most significant association with all offspring outcomes (results available from F.R.). This is consistent with findings from prospective studies and our prediction that late pregnancy stress was likely to have the strongest effects on offspring psychopathology in line with recent literature reviews (Rice et al. 2007; Talge et al. 2007). For simplicity and to avoid multiple testing, we focus the rest of our analyses on this measure of pregnancy stress.

\section{Prenatal stress and birth weight}

Maternal report of late pregnancy stress was significantly associated with lower infant birth weight when the mother was unrelated to the child $(b=$ $-43.53, \beta=-0.189, p=0.01$ ) (adjusting for covariates). A similar reduction in birth weight was found when the mother was related to the child $(b=-29.37$, $\beta=-0.127, p=0.002)$. These results remained significant $(\beta=-0.221, p=0.01 ; \beta=-0.118, p=0.02$, respectively) when the analysis was confined to singleton pregnancies. Consistent with an environmental effect, when testing for differences in the magnitude of association between prenatal stress and birth weight for each group (related; unrelated), the interaction term was not significant $(\beta=0.066, p=$ 0.377).

\section{Prenatal stress and gestational age}

There were significant associations between late prenatal stress and lower infant gestational age in unrelated $(\beta=-0.265, p=0.001)$ and related $(\beta=-0.209$, $p=0.001)$ pregnancies. Thus, results are consistent with environmental influences of prenatal stress on infant gestational age. Consistent with this, the interaction effect between prenatal stress and group (related; unrelated) was not significant $(\beta=0.057$, $p=0.474)$.

\section{Prenatal stress and child mental health}

\section{Child antisocial behaviour}

Prenatal stress showed significant association with child antisocial behaviour in the unrelated mothers $(\beta=0.190, p=0.02)$ (Table 3). A significant association was also found in the related mother-child dyads $(\beta=0.186, p=0.001)$. Thus, the association seems to be attributable to environmental influences. Consistent with this, the interaction was not significant $(\beta=$ $-0.050, p=0.552$ ).

\section{Child anxiety}

There were significant associations between prenatal stress and offspring anxiety in the unrelated motherchild pairs $(\beta=0.211, p=0.01)$ and those that were related $(\beta=0.207, p=0.001)$. Thus association appears to be due to environmental pathways. Consistent with this, the interaction term was not significant $(\beta=0.073$, $p=0.361$ ).

\section{Child ADHD}

No significant association was found between prenatal stress and offspring ADHD in the group of mothers who were unrelated to their child $(\beta=0.095, p=0.25)$. There was a significant association only in the group of mothers that were related to their child $(\beta=0.163$, $p=0.001)$. This association therefore appeared attributable to maternally inherited factors. Consistent with this, when testing for differences across the related and unrelated groups in the association between 
Table 2. Intercorrelations between prenatal stress, perinatal factors, maternal characteristics and child adjustment

\begin{tabular}{|c|c|c|c|c|c|c|c|c|c|c|c|c|c|c|c|c|}
\hline & 1 & 2 & 3 & 4 & 5 & 6 & 7 & 8 & 9 & 10 & 11 & 12 & 13 & 14 & 15 & 16 \\
\hline 1 Child gender $^{\mathrm{a}}$ & - & & & & & & & & & & & & & & & \\
\hline 2 Child age & -0.042 & - & & & & & & & & & & & & & & \\
\hline 3 Child anxiety symptoms & -0.053 & $0.111^{* *}$ & - & & & & & & & & & & & & & \\
\hline 4 Child antisocial behaviour & -0.057 & $-0.076^{*}$ & $0.345^{* *}$ & - & & & & & & & & & & & & \\
\hline 5 Child ADHD symptoms & $-0.152^{* *}$ & -0.030 & $0.502^{* *}$ & $0.505^{* *}$ & - & & & & & & & & & & & \\
\hline 6 Prenatal stress early & 0.058 & 0.027 & $0.133^{* *}$ & $0.072^{*}$ & $0.080^{*}$ & - & & & & & & & & & & \\
\hline 7 Prenatal stress mid & -0.009 & -0.005 & $0.216^{* *}$ & $0.161^{* *}$ & $0.121^{* *}$ & $0.621^{* *}$ & - & & & & & & & & & \\
\hline 8 Prenatal stress late & 0.020 & -0.068 & $0.180^{* *}$ & $0.173^{* *}$ & $0.121^{* *}$ & $0.325^{* *}$ & $0.595^{* *}$ & - & & & & & & & & \\
\hline $\begin{array}{l}9 \text { Mother current anxiety/ } \\
\text { depression }\end{array}$ & -0.003 & 0.002 & $0.347^{* *}$ & $0.245^{* *}$ & $0.254^{* *}$ & $0.231^{* *}$ & $0.317^{* *}$ & $0.279^{* *}$ & - & & & & & & & \\
\hline 10 Mother age at childbirth & 0.026 & -0.057 & -0.060 & -0.016 & -0.025 & 0.041 & 0.038 & -0.022 & 0.028 & - & & & & & & \\
\hline 11 Mother height & -0.005 & 0.031 & 0.011 & -0.050 & 0.005 & -0.017 & 0.001 & -0.003 & 0.011 & -0.060 & - & & & & & \\
\hline 12 Child birth weight & -0.020 & -0.025 & -0.054 & -0.044 & -0.021 & -0.026 & $-0.178^{* *}$ & $-0.258^{* *}$ & -0.034 & 0.024 & $0.089 *$ & - & & & & \\
\hline $\begin{array}{l}13 \text { Antenatal complications } \\
\text { composite }\end{array}$ & $-0.074^{*}$ & -0.011 & 0.033 & 0.042 & 0.049 & $0.096^{* *}$ & $0.177^{* *}$ & $0.212^{* *}$ & $0.082^{*}$ & $0.079^{*}$ & -0.050 & $-0.423^{* *}$ & - & & & \\
\hline 14 Socio-occupational status & 0.006 & -0.049 & 0.060 & $0.145^{* *}$ & $0.109^{* *}$ & -0.046 & 0.055 & 0.013 & $0.086^{*}$ & -0.178 & -0.026 & $-0.074^{*}$ & -0.022 & - & & \\
\hline 15 Gestational age & 0.051 & -0.006 & -0.047 & -0.052 & -0.015 & -0.041 & $-0.179^{* *}$ & $-0.299^{* *}$ & -0.041 & -0.029 & 0.011 & $0.759^{* *}$ & $-0.387^{* *}$ & -0.005 & - & \\
\hline 16 Family income & -0.026 & -0.010 & $-0.081^{*}$ & $-0.074^{*}$ & -0.066 & 0.036 & -0.011 & 0.003 & $-0.122^{* *}$ & $0.178^{* *}$ & 0.032 & 0.014 & 0.064 & $-0.618^{* *}$ & -0.030 & - \\
\hline
\end{tabular}

ADHD, Attention deficit hyperactivity disorder.

${ }^{a}$ Child gender was coded $1=$ male, $2=$ female.

${ }^{*} p<0.05,{ }^{* *} p<0.01$. 
Table 3. Association between late prenatal stress and child psychopathology in related and unrelated offspring ${ }^{\mathrm{a}, \mathrm{b}}$

\begin{tabular}{|c|c|c|c|c|}
\hline & Child outcome & $\begin{array}{l}\text { Prenatal stress, } \\
\text { standardized } \beta\end{array}$ & $\begin{array}{l}\text { Difference between } \\
\text { unrelated and related } \\
\text { groups, standardized } \beta\end{array}$ & $\begin{array}{l}\text { Prenatal stress controlling } \\
\text { for post-natal anxiety / } \\
\text { depression, standardized } \beta\end{array}$ \\
\hline Unrelated offspring & Anxiety symptoms & $0.211^{* *}$ & 0.073 & 0.114 \\
\hline Related offspring & Anxiety symptoms & $0.207^{* * *}$ & & $0.119^{* *}$ \\
\hline Unrelated offspring & Antisocial behaviour & $0.186^{* * *}$ & -0.050 & $0.171^{*}$ \\
\hline Related offspring & Antisocial behaviour & $0.190^{* *}$ & & $0.102^{*}$ \\
\hline Unrelated offspring & ADHD symptoms & 0.095 & $0.157^{*}$ & - \\
\hline Related offspring & ADHD symptoms & $0.163^{* * *}$ & & - \\
\hline
\end{tabular}

ADHD, Attention deficit hyperactivity disorder.

${ }^{a}$ Coefficients are adjusted for child gender, child age, antenatal factors and social occupational class.

${ }^{\mathrm{b}}$ When an association between prenatal stress and offspring outcome appeared to be due to environmental effects, we tested if the association could be attributable to post-natal anxiety/depression by including this variable as an additional covariate. The association between prenatal stress and offspring ADHD appeared to be due to a genetic association.

${ }^{*} p<0.05,{ }^{* *} p<0.01,{ }^{* * *} p<0.001$.

prenatal stress and ADHD, the interaction term was significant $(\beta=0.157, p=0.05)$.

\section{Including current maternal anxiety and depression symptoms as a covariate}

After identifying associations that could be attributed to environmental factors, we examined the possible role of the 'post-natal environment' by including current maternal anxiety/depression symptoms as a covariate in addition to the other covariates described. We did not further examine the association between prenatal stress and offspring ADHD because this appeared to be attributable to inherited rather than environmental factors. As expected, including maternal anxiety/depression made no difference to the pattern of results for offspring birth weight $(\beta=$ $-0.145, p=0.001$ unrelated; $\beta=-0.197, p=0.01$ related) and gestational age $(\beta=-0.265, p=0.001$ unrelated; $\beta=-0.209, p=0.001$ related).

Table 3 shows that including current maternal anxiety/depression made no difference to the pattern of results for offspring antisocial behaviour $(\beta=0.171$, $p=0.04$ unrelated; $\beta=0.102, p=0.02$ related). However, for offspring anxiety, the pattern of results changed whereby a significant association was now observed only in the related group $(\beta=0.114, p=0.145$ unrelated; $\beta=0.119, p=0.008$ related). The association of current maternal anxiety/depression with child anxiety was also environmentally linked in that associations were significant in women who were unrelated to their child $(\beta=0.351, p=0.001)$ and those who were related $(\beta=0.347, p=0.001)$ - suggesting a post-natal environmental influence. We formally tested whether current maternal anxiety/depression was mediating the association between prenatal stress and child anxiety. Current maternal anxiety/depression was found to significantly mediate the association between late prenatal stress and child anxiety in the whole sample (Sobel statistic $=6.11, p<0.00001$ ) as well as in the unrelated (Sobel statistic $=2.48, p=0.01$ ) and related (Sobel statistic $=5.44, p<0.00001$ ) groups respectively. This confirms that the initially observed environmental association (reported in the main results section) was confounded with influences of current maternal anxiety/depression.

\section{Gender differences}

No gender differences were observed in the patterns of association between prenatal stress and child outcomes.

\section{Discussion}

Our findings suggest that, with the exception of offspring ADHD, associations between prenatal stress and the offspring outcomes we examined, that have been identified in previous prospective studies, cannot be attributed to inherited factors transmitted from mother to child. In contrast to ADHD, associations between prenatal stress and offspring birth weight, gestational age and antisocial behaviour were observed in both unrelated and related mother-child dyads. In addition, our data suggested that taking into account current maternal anxiety/depression changed the findings for the relationship between prenatal stress and offspring anxiety such that current maternal anxiety/depression accounted for the link between prenatal stress and offspring anxiety.

Our first set of findings relates to the relationship between prenatal stress and offspring birth weight and 
gestational age. Psychosocial stress during pregnancy is known to result in offspring birth-weight reductions (Lederman et al. 2004; Borders et al. 2007; Rahman et al. 2007). Animal studies and clinical observations of infants who have been exposed to synthetic glucocorticoids suggest that prenatal stress in pregnancy and stress hormones reduce offspring birth weight through environmental mechanisms (Guinn et al. 2001; Aghajafari et al. 2002). Calorific intake and epigenetic changes may also impact upon offspring birth weight (Gluckman et al. 2007). However, it has not been possible to show in humans that these associations are necessarily environmental effects of stress. Given that perception of stress is highly heritable (Kendler \& Prescott, 2006) it is difficult to rule out the possibility that associations between prenatal stress and outcomes in naturalistic studies of humans are due to inherited factors. Our study suggests that this explanation for the link between prenatal stress and lower birth weight is unlikely. Finally, prenatal stress also showed significant association with lower gestational age and this too was not due to inherited factors. This finding is consistent with previous evidence that elevated levels of maternal stress hormones are associated with both reduced birth weight and premature onset of labour (Wadhwa et al. 1998; Aghajafari et al. 2002).

Our second set of findings relates to links between prenatal stress and child mental health outcomes. Many population surveys have shown that maternal anxiety/stress during pregnancy is associated with increased emotional and antisocial problems in the children even when controlling for important confounders (Rice et al. 2007; Talge et al. 2007; Swanson \& Wadhwa, 2008). This has increasingly led to the conclusion that exposure to prenatal stress has causal risk effects on psychopathology but it is not clear if these findings are due to genetic influences, as psychopathology is heritable (Rutter et al. 1999). Our results showed that the link between prenatal stress and offspring ADHD did appear to be due to inherited factors in that association was not found in the unrelated group. These findings argue against prenatal stress having risk effects on offspring ADHD. In contrast, for other child mental health outcomes, we did find evidence for environmental links with prenatal stress. For antisocial behaviour and anxiety, association was found in both unrelated and related offspring.

After identifying associations between prenatal stress and offspring mental health outcomes that were not attributable to inherited pathways, we included current maternal anxiety/depression as a covariate. Results did not change for offspring antisocial behaviour (or birth weight and gestational age), but did for offspring anxiety. These results highlight the importance of separating pre- and post-natal effects of anxiety/depression symptoms in future prospective studies (Swanson \& Wadhwa, 2008) and are consistent with findings from some cross-fostering animal studies showing that prenatal stress can influence offspring behaviour indirectly via changes to the postnatal rearing environment (Maccari et al. 1995; Pardon et al. 2000; Champagne \& Meaney, 2006).

Notwithstanding the limitations inherent to any cross-sectional design, it should be highlighted that this sample is unique in several respects. Despite homologous IVF treatment being relatively common, other treatments are infrequent; for example, approximately $6 \%$ of IVF cycles performed every year in the UK involve the use of donated oocytes and fewer still use donated embryos (Adamson et al. 2006). There is a shortage of altruistic egg donors in the UK and our design only included donors who were unrelated to the couple undergoing treatment, hence this also added to the difficulty in obtaining the sample. Nevertheless, it should be noted that other genetically informative designs such as the children of twins design are helpful for disentangling the contribution of inherited and prenatal factors (e.g. Knopick et al. 2005, 2006) although to date prenatal stress has not been examined using this design.

Using this new quasi-experimental design, three factors may be important. First, could the foetus's genes influence the prenatal environment in the unrelated group? Although this is possible, it seems unlikely to account for our results because, in the unrelated group, the foetus's genes will be inherited in a random fashion from unrelated oocyte/embryo donors. Also, for prenatal stress, the effects of foetal genes would presumably be mediated through antenatal complications and these were included as covariates. Second, epigenetic effects are thought to be important. In this design, these will emerge as environmental effects to the extent that epigenetic changes can be induced by environmental modification and stochastic processes (Fraga et al. 2005; Feinberg, 2007). Third, we controlled for potential confounding factors by including them as covariates. This will necessarily remove inherited and environmental variation in predictor and outcome variables although not in a systematic way.

\section{Limitations}

There may be some concern about whether our results are generalizable given that these children were conceived using IVF. However, studies have consistently shown that children conceived via IVF are similar in terms of psychological adjustment to naturally conceived children (Golombok \& MacCallum, 2003; Tully 
et al. 2003; Barnes et al. 2004). The present sample is also comparable with population-based data in terms of child and parent mental health and family income (Thapar et al. 2007; Shelton et al. in press) although it is not representative in terms of antenatal risk (Thapar et al. 2007). Nonetheless, the sample includes families from a range of demographic backgrounds (IVF treatment is freely provided by the National Health Service in the UK given certain eligibility criteria). Moreover, all comparisons of association were conducted between mothers who conceived via IVF; therefore, groups were similar in terms of method of conception and having a history of fertility problems. It is possible that women who conceive using IVF report higher levels of prenatal stress than women who conceive naturally. Levels of global anxiety during pregnancy are similar between women who conceive via IVF and those who conceive naturally, although levels of anxiety about the health of the unborn child appear elevated in women undergoing IVF (McMahon et al. 1997). It seems possible that a history of difficult or failed pregnancies may increase perceptions of prenatal stress. However, we did not find evidence of this. For example, women with a history of miscarriage $(n=77)$ did not report higher levels of prenatal stress $[t(446)=-0.391, p=0.696]$. Another limitation is that our measure of prenatal stress was by necessity maternally rated and retrospective and that women conceiving via IVF with egg or embryo donation reported slightly higher rates of prenatal stress. As mothers reported on both prenatal stress and child outcome, shared method variance may be a limiting factor. Nevertheless, we were able to take into account current maternal anxiety/depression and our results highlighted the importance of doing so, as findings for child anxiety changed when adjustments were made for 'post-natal' anxiety/depression. Also, our measure of prenatal stress showed association with multiple measures of infant size at birth as assessed by antenatal records, thus providing evidence of construct validity. The relatively wide age range of the children in the study (4-10 years) may mean that there were developmental differences in childhood psychopathology. Finally, this genetically informative design can detect inherited effects and can demonstrate consistency with environmental links between prenatal risk factors and offspring outcomes; however, it cannot demonstrate causality and we did not consider other post-natal variables that could potentially moderate or mediate associations between prenatal stress and offspring outcomes.

In summary, we find that associations between prenatal stress with reduced birth weight, gestational age and with increased antisocial behaviour appear to be due to environmental factors, suggesting that future studies of prenatal stress using alternative research designs could usefully focus on these offspring outcomes. In contrast, for offspring ADHD symptoms, the association was due to genetic factors inherited by offspring from mothers, since association was not seen in unrelated mother-child pairs. Our results for offspring anxiety highlight the importance of testing for timing effects of prenatal and post-natal maternal stress in prospective studies.

Appendix 1. Correlation matrix between stages of prenatal stress in test-retest analysis

\begin{tabular}{|c|c|c|c|c|c|}
\hline & 1 & 2 & 3 & 4 & 5 \\
\hline 1 Early stress & - & & & & \\
\hline $2 \mathrm{Mid}$ stress & $0.566^{* *}$ & - & & & \\
\hline 3 Late stress & $0.213^{*}$ & $0.587^{* *}$ & - & & \\
\hline $\begin{array}{l}4 \text { Early stress } \\
\text { retest }\end{array}$ & $0.734^{* *}$ & $0.343^{* *}$ & 0.180 N.s. & - & \\
\hline $\begin{array}{l}5 \text { Mid stress } \\
\text { retest }\end{array}$ & $0.429^{* *}$ & $0.723^{* *}$ & $0.467^{* *}$ & $0.520^{* *}$ & - \\
\hline $\begin{array}{l}6 \text { Late stress } \\
\text { retest }\end{array}$ & $0.230^{*}$ & $0.584^{* *}$ & $0.705^{* *}$ & $0.326^{* *}$ & $0.620^{* *}$ \\
\hline
\end{tabular}

\section{Acknowledgements}

This project has been funded by a Wellcome Trust Showcase Award and a Wellcome Trust Project grant. We thank Val Russell for administrative support and Allyson Lewis for collecting antenatal data. Thanks also to Xiaojia Ge for contributions to the study design and to Michael Owen and Michael Rutter for comments on an earlier draft of the paper. We are extremely grateful to our collaborators at the following fertility centres: Assisted Reproduction Unit, Aberdeen University; Boston IVF, Boston, USA; Bourn Hall Clinic; Bridgewater Hospital, Manchester; Cardiff Assisted Reproduction Unit; CARE at Sheffield Fertility Centre; Centre for Reproductive Medicine, University of Bristol; Centre for Reproductive Medicine, Walsgrave Hospital, Coventry; Childlessness Overcome Through Surrogacy; Clarendon Wing, Leeds General Infirmary; Assisted Conception Unit, St James' Hospital, Leeds; Cromwell IVF and Fertility Centre, London; Edinburgh Royal Infirmary; The Hewitt Centre for Reproductive Medicine Unit, Liverpool Women's Hospital; London Fertility and Gynaecology Centre; London Women's Clinic; Midland Fertility Services; St Mary's Hospital Regional IVF and DI (donor insemination) Unit, Manchester. In addition, we thank all the families who have generously participated in this study. 


\section{Declaration of Interest}

None.

\section{References}

Adamson GD, de Mouzon J, Lancaster P, Nygren KG, Sullivan E, Zegers-Hochschild F (2006). World collaborative report on in vitro fertilization, 2000. Fertility and Sterility 85, 1586-1622.

Aghajafari F, Murphy K, Matthews S, Ohlsson A, Amankwah K, Hannah M (2002). Repeated doses of antenatal corticosteroids in animals: a systematic review. American Journal of Obstetrics and Gynecology 186, 843-849.

APA (1994). Diagnostic and Statistical Manual of Mental Disorders, 4th edn, revised. American Psychological Association: Washington, DC.

Barnes J, Sutcliffe AG, Kristoffersen I, Loft A, Wennerholm U, Tarlatzis BC, Kantaris X, Nekkebroeck J, Hagberg BS, Madsen SV, Bonduelle M; European Study (2004). The influence of assisted reproduction on family functioning and children's socio-emotional development: results from a European study. Human Reproduction 19, 1480-1487.

Baron RM, Kenny DA (1986). The moderator-mediator variable distinction in social psychological research: conceptual, strategic, and statistical considerations. Journal of Personality and Social Psychology 51, 1173-1182.

Barrett H (2006). Attachment and the Perils of Parenting. National Family and Parenting Institute: London.

Borders AE, Grobman WA, Amsden LB, Holl JL (2007). Chronic stress and low birthweight neonates in a low-income population of women. Obstetrics and Gynecology 109, 331-338.

Champagne FA, Meaney MJ (2006). Stress during gestation alters postpartum maternal care and the development of the offspring in a rodent model. Biological Psychiatry 59, 1227-1235.

Chang HL, Chang TC, Lin TY, Kuo SS (2002). Psychiatric morbidity and pregnancy outcome in a disaster area of Taiwan 921 earthquake. Psychiatry and Clinical Neurosciences 56, 139-144.

DuPaul GJ (1981). Parent and teacher ratings of ADHD symptoms: psychometric properties in a community-based sample. Journal of Clinical Child Psychology 20, 245-253.

Feinberg AP (2007). Phenotypic plasticity and the epigenetics of human disease. Nature 447, 433-440.

Fraga MF, Ballestar E, Paz MF, Ropero S, Setien F, Ballestar ML, Heine-Suñer D, Cigudosa JC, Urioste $M$, Benitez J, Boix-Chornet M, Sanchez-Aguilera A, Ling C, Carlsson E, Poulsen P, Vaag A, Stephan Z, Spector TD, Wu YZ, Plass C, Esteller M (2005). Epigenetic differences arise during the lifetime of monozygotic twins. Proceedings of the National Academy of Sciences USA 102, 10604-10609.

Gluckman PD, Hanson MA, Beedle AS (2007). Nongenomic transgenerational inheritance of disease risk. BioEssays 29, 145-154.
Golombok S, MacCallum F (2003). Practitioner review: outcomes for parents and children following nontraditional conception: what do clinicians need to know? Journal of Child Psychology and Psychiatry 44, 303-315.

Goodman R (1997). The Strengths and Difficulties Questionnaire: a research note. Journal of Child Psychology and Psychiatry 38, 581-586.

Guinn DA, Atkinson MW, Sullivan L, Lee M, MacGregor S, Parilla BV, Davies J, Hanlon-Lundberg K, Simpson L, Stone J, Wing D, Ogasawara K, Muraskas J (2001). Single vs weekly courses of antenatal corticosteroids for women at risk of preterm delivery: a randomized controlled trial. Journal of the American Medical Association 286, 1581-1587.

Huizink AC, Mulder EJH, Buitelaar JK (2004). Prenatal stress and risk for psychopathology: specific effects or induction of general susceptibility? Psychological Bulletin 130, 115-142.

Kendler KS, Prescott CA (2006). Genes, Environment and Psychopathology: Understanding the Causes of Psychiatric and Substance Use Disorders. The Guilford Press: London.

Knopick VS, Heath AC, Jacob T, Slutske WS, Bucholz KK, Madden PA, Waldron M, Martin NG (2006). Maternal alcohol use disorder and offspring ADHD: disentangling genetic and environmental effects using a children-of-twins design. Psychological Medicine 36, 1461-1471.

Knopick VS, Sparrow EP, Madden PA, Bucholz KK, Hudziak JJ, Reich W, Slutske WS, Grant JD, McLaughlin TL, Todorov A, Todd RD, Heath AC (2005). Contributions of parental alcoholism, prenatal substance exposure and genetic transmission to child ADHD risk: a female twin study. Psychological Medicine 35, 625-635.

Lederman SA, Rauh V, Weiss L, Stein JL, Hoepner LA, Becker M, Perera FP (2004). The effects of the World Trade Center event on birth outcomes among deliveries at three lower Manhattan hospitals. Environmental Health Perspectives 112, 1772-1778.

Maccari S, Liazza PV, Kabbaj M, Barbazanges A, Simon H, Le Moal M (1995). Adoption reverses the long-term impairment in glucocortoid feedback induced by prenatal stress. Journal of Neuroscience 15, 110-115.

Maughan B, Taylor A, Caspi A, Moffitt TE (2004). Prenatal smoking and early childhood conduct problems: testing genetic and environmental explanations of the association. Archives of General Psychiatry 61, 836-843.

McMahon CA, Ungerer JA, Beaurepaire J, Tennant C, Saunders D (1997). Anxiety during pregnancy and fetal attachment after in-vitro fertilization conception. Human Reproduction 12, 176-182.

Nygren KG, Andersen AN (1999). Assisted reproductive technology in Europe, 1999: results generated from European registers by ESHRE. Human Reproduction 17, 3260-3274.

O'Connor TG, Heron J, Glover V, ALSPAC study team (2002a). Antenatal anxiety predicts child behavioural/ emotional problems independently of postnatal 
depression. Journal of the American Academy of Child and Adolescent Psychiatry 41, 1470-1477.

O'Connor TG, Heron J, Golding J, Beveridge M, Glover V (2002b). Maternal antenatal anxiety and children's behavioural/emotional problems at 4 years. British Journal of Psychiatry 180, 502-508.

O'Connor TG, Heron J, Golding J, Glover V, ALSPAC study team (2003). Maternal antenatal anxiety and behavioural/emotional problems in children: a test of a programming hypothesis. Journal of Child Psychology and Psychiatry 44, 1025-1036.

Office for National Statistics (2000). Standard Occupational Classification. The Stationery Office: London.

Pardon M, Gerardin P, Joubert C, Perez-Diaz F, Cohen-Salmon C (2000). Influence of prepartum chronic ultramild stress on maternal pup care behaviour in mice. Biological Psychiatry 47, 858-863.

Preacher KJ, Leonardelli GJ (2001). Calculation for the Sobel Test. An interactive calculation tool for mediation tests (http://www.people.ku.edu/ preacher/sobel/sobel. htm). Accessed 1 August 2008.

Rahman A, Bunn J, Lovel H, Creed F (2007). Association between antenatal depression and low birthweight in a developing country. Acta Psychiatrica Scandinavica 115, 481-486.

Rice F, Harold GT, Boivin J, Hay D, van den Bree MMB, Thapar A (2009). Disentangling prenatal and inherited influences in humans with an experimental design. Proceedings of the National Academy of Sciences USA 106, 2464-2467.

Rice F, Jones I, Thapar A (2007). The impact of gestational stress and prenatal growth on emotional problems in offspring: a review. Acta Psychiatrica Scandinavica 115, 171-183.

Rice F, Lewis A, Harold G, van den Bree M, Boivin J, Hay DF, Owen MJ, Thapar A (2007). Agreement between maternal report and antenatal records for a range of pre and peri-natal factors: the influence of maternal and child characteristics. Early Human Development 83, 497-504.

Rutter M (2007). Proceeding from observed correlation to causal inference: the use of natural experiments. Perspectives on Psychological Science 2, 377-395.

Rutter M, Pickles A, Murray R, Eaves L (2001). Testing hypotheses on specific environmental causal effects on behaviour. Psychological Bulletin 127, 291-324.

Rutter M, Silberg J, O'Connor T, Simonoff E (1999). Genetics and child psychiatry: II. Empirical research findings. Journal of Child Psychology and Psychiatry 40, 19-55.

Sandman CA, Wadhwa PD, Chicz-DeMet A, DunkelSchetter C, Porto M (1997). Maternal stress, HPA activity and fetal/infant outcome. Neuropeptides in Development and Aging 814, 266-275.

Shelton KH, Rice F, Harold GT, Hay D, Boivin J, van den Bree MBM, Thapar A (in press). Examining differences in psychological adjustment problems among children conceived by assisted reproductive technologies. International Journal of Behavioral Development.

Swanson JS, Wadhwa PM (2008). Developmental origins of child mental health disorders. Journal of Child Psychology and Psychiatry 49, 1009-1019.

Talge NM, Neal C, Glover V, Early Stress Translational Research Prevention Science Network (2007). Fetal and neonatal experience on child and adolescent mental health. Journal of Child Psychology and Psychiatry 48, 245-261.

Thapar A, Harold GT, Rice F, Ge X, Boivin J, Hay D, van den Bree M, Lewis A (2007). Do intrauterine or genetic influences explain the fetal origins of chronic disease? A novel experimental method for disentangling effects. BMC Medical Research Methodology 7, 25.

Tully LA, Moffitt T, Caspi A (2003). Maternal adjustment, parenting and child behaviour in families of school-aged twins conceived after IVF and ovulation induction. Journal of Child Psychology and Psychiatry 44, 316-325.

Valero de Bernabe J, Soriano T, Albaladejo R, Juarranz M, Calle ME, Martínez D, Domínguez-Rojas V (2004). Risk factors for low birth weight: a review. European Journal of Obstetrics, Gynaecology and Reproductive Biology 116, 3-15.

van Den Bergh BR, Marcoen A (2004). High antenatal maternal anxiety is related to ADHD symptoms, externalizing problems and anxiety in 8 and 9 year olds. Child Development 75, 1085-1097.

Wadhwa PD, Porto M, Garite TJ, Chicz-DeMet A, Sandman CA (1998). Maternal corticotropin releasing hormone levels in the early third trimester predict length of gestation in human pregnancy. American Journal of Obstetrics and Gynecology 179, 10791085.

Wadhwa PD, Sandman CA, Porto M, Dunkelschetter C, Garite TJ (1993). The association between prenatal stress and infant birth weight and gestational age and birth - a prospective investigation. American Journal of Obstetrics and Gynecology 169, 858-865.

Weinstock M (2005). The potential influence of maternal stress hormones on development and mental health of the offspring. Brain, Behaviour and Immunity 19, 296-308.

Welberg LA, Seckl JR (2001). Prenatal stress, glucocorticoids and the programming of the brain. Journal of Neuroendocrinology 13, 113-128.

Whittinger NS, Langley K, Fowler TA, Thomas HV, Thapar A (2007). Clinical precursors of adolescent conduct disorder in children with attention-deficit/ hyperactivity disorder. Journal of the American Academy of Child and Adolescent Psychiatry 46, 179-187.

Zigmond AS, Snaith RP (1983). The Hospital Anxiety and Depression Scale. Acta Psychiatrica Scandinavica 67, 361-370. 